\title{
TITLE
}

\section{Effect of cocoa's theobromine on intestinal microbiota of rats}

\section{AUTHORS}

Sandra Martín-Peláez ${ }^{1,2, *}$, Mariona Camps-Bossacoma ${ }^{2,3}$, Malen Massot-Cladera ${ }^{2,3}$, Mar Rigo-Adrover $^{2,3}$, Àngels Franch ${ }^{2,3}$, Francisco J Pérez-Cano ${ }^{2,3}$, Margarida Castell ${ }^{2,3}$

\section{AFFILIATIONS}

${ }^{1}$ Cardiovascular Risk and Nutrition Research Group, REGICOR Study Group. Hospital del Mar Research Institute (IMIM), Barcelona, Spain. Spanish Biomedical Research Networking Centre-Physiopathology of Obesity and Nutrition (CIBERobn), Health Institute Carlos III, Madrid, Spain

${ }^{2}$ Physiology Section, Department of Biochemistry and Physiology, Faculty of Pharmacy and Food Science, University of Barcelona (UB), Barcelona, Spain

${ }^{3}$ Nutrition and Food Safety Research Institute (INSA-UB), Santa Coloma de Gramenet, Spain

\section{KEYWORDS}

FISH; metagenomics; methylxanthines; microbiota; short-chain fatty acids

\section{CORRESPONDENCE*}

Dr Sandra Martín-Peláez, s.martin.pelaez@gmail.com 


\section{ABBREVIATIONS}

CC: cocoa

F/B: Firmicutes/Bacteroidetes ratio

FBS: fetal bovine serum

FCM: flow cytometry

FISH: fluorescence in situ hybridization

OTU: operational taxonomic units

PI: propidium iodide

RF: reference

TB: theobromine 


\section{ABSTRACT}

2 SCOPE: To establish the role of cocoa theobromine on gut microbiota composition and

3 fermentation products after cocoa consumption in rats.

4 METHODS AND RESULTS: Lewis rats were fed either a standard diet (RF diet), a diet

5 containing $10 \%$ cocoa (CC diet) or a diet including $0.25 \%$ theobromine (TB diet) for 15 days.

6 Gut microbiota (fluorescence in situ hybridization coupled to flow cytometry and

7 metagenomics analysis), SCFA and IgA-coated bacteria were analyzed in fecal samples.

$8 \mathrm{CC}$ and TB diets induced lower counts of E. coli whereas TB diet led to lower counts of

9 Bifidobacterium spp., Streptococcus spp. and Clostridium histolyticum-C. perfingens group

10 compared to RF diet. Metagenomics analysis also revealed a different microbiota pattern

11 among the studied groups. The SCFA content was higher after both CC and TB diets, which

12 was mainly due to enhanced butyric acid production. Furthermore, both diets decreased the

13 proportion of $\operatorname{IgA}$-coated bacteria.

14 CONCLUSION: Cocoa's theobromine plays a relevant role in some effects related to cocoa

15 intake, such as the lower proportion of IgA-coated bacteria. Moreover, theobromine modifies

16 gut microbiota although other cocoa compounds could also act on intestinal bacteria,

17 attenuating or enhancing the theobromine effects. 


\section{Introduction}

20 Although cocoa powder was initially used for medical purposes by Mesoamerican civilizations [1], it is only recently that cocoa has come to be considered a valuable product with healthy properties [2]. Among these beneficial effects, it has been reported that cocoaenriched diets modulate the immune system and the gut microbiota [3]. In particular, a cocoaenriched diet is able to attenuate secretory $\operatorname{IgA}(\mathrm{S}-\operatorname{Ig} \mathrm{A})$ in several intestinal compartments [46] and also the IgA-coated bacteria proportion in the gut [5]. Moreover, a diet containing 10\% cocoa for 6 weeks modifies the intestinal microbiota composition in rats by decreasing the proportion of the Bacteroides spp., the Staphylococcus spp., and the Clostridium histolyticum subgroup [5], and thus causing a different short-chain fatty acid (SCFA) production [7]. Similarly, a cocoa diet modulates the intestinal microbiota in orally sensitized rats, as determined by a metagenomics analysis [8].

Cocoa powder contains macronutrients, fiber, minerals, polyphenols (flavonoids, mainly flavanols) and methylxanthines [9]. The most abundant xanthine found in cocoa is theobromine, followed by caffeine. In fact, cocoa is the richest natural source of theobromine $[10,11]$. While the effects of flavonoids present in cocoa have been thoroughly studied, less attention has been paid to the presence of theobromine in cocoa. Even so, a few studies have related its content to a variety of properties attributed to cocoa powder [10, 12]. As theobromine is able to reach the gut $[13,14]$, we hypothesized that this methylxanthine could contribute to the effects of cocoa intake on gut microbiota. Therefore, the purpose of the present work was to establish the role of cocoa theobromine in the composition of gut microbiota and fermentation products after cocoa consumption in rats. 


\section{Material and methods}

\subsection{Animals and diets}

Lewis rats (3 week old) obtained from Janvier Labs (Saint-Berthevin Cedex, France) were housed in cages (2-3 animals/cage on days $0-8$, and individually on days $8-15$ ) under controlled temperature and humidity in a 12:12 h light:dark cycle. The rats were randomly distributed into three dietary groups ( $\mathrm{n}=7$ per group): the reference (RF) group ingested a standard diet AIN-93M (Teklad, Madison, USA), the cocoa (CC) group ingested a standard diet with $10 \%$ of natural Forastero cocoa (Idilia Foods S.L., Barcelona, Spain) containing $2.5 \%$ theobromine, and the theobromine (TB) group ingested a standard diet including $0.25 \%$ of theobromine (Sigma-Aldrich, Madrid, Spain), i.e. the content of theobromine present in the CC diet. The two experimental diets were elaborated on the basis of the AIN-93M formula by subtracting the amount of carbohydrates, proteins, lipids and insoluble fiber provided by the corresponding supplements. The resulting diets were isoenergetic and contained the same proportion of macronutrients and insoluble fiber as the RF diet (Table 1). Animals were provided with feed and water ad libitum for 2 weeks. Animal procedures were approved by the Ethical Committee for Animal Experimentation of the University of Barcelona (ref. 5988).

\subsection{Fecal samples collection and pre-analytical procedures}

Fresh feces were collected at days 0,8 and 15 and processed according to the specific variables to be analyzed. Some fresh fecal samples were used to determine fecal $\mathrm{pH}$, using a surface electrode (Crison Instruments, S.A., Barcelona, Spain). The rest of the fecal samples were directly frozen either at $-20^{\circ} \mathrm{C}$ until the metagenomics analysis, the bacterial characterization by fluorescence in situ hybridization, and the IgA-coated bacteria quantification, or at $-80^{\circ} \mathrm{C}$ until SCFA analysis. For these determinations, fecal homogenates were later obtained following procedures previously described [5]. 
2.3. Quantification of fecal microbiota by fluorescence in situ hybridization (FISH) coupled to flow cytometry (FCM)

Quantification of representative groups of gut microbiota was carried out in feces from day 15 by FISH coupled to FCM (FISH-FCM), as described by Massot-Cladera et al. [15]. Briefly, fixed fecal suspensions were incubated with Cy5-labeled probes targeting specific diagnostic regions of 16S rRNA from different gut bacterial groups (Bacteroidaceae-Prevotellaceae group, Bac303; Bifidobacterium spp., Bif164; Clostridium histolyticum-C. perfringens group,

Chis150; Escherichia coli, Ec1531; Clostridium coccoides-Eubacterium rectale group,

Erec482; Lactobacillus-Enterococcus group, Lab158, Staphylococcus spp., Staphy;

Streptococcus spp., Strept) (Supplementary Table 1). In the case of Lactobacillus, samples were permeabilized with lysozyme (Serva, Heidelberg, Germany) prior to the hybridization process [16]. All samples were hybridized at the specific probe hybridization temperature, as described [15], and kept in the dark at $4{ }^{\circ} \mathrm{C}$ overnight until FCM analysis.

To determine the total bacteria number, the samples were mixed with propidium iodide (PI, $1 \mathrm{mg} / \mathrm{mL}$; Sigma-Aldrich, Madrid, Spain) prior to FCM analysis [5].

\subsection{Determination of the proportion of bacteria coated with $\operatorname{IgA}$}

Quantification of IgA-coated bacteria was carried out as previously described [15].

\subsection{Flow cytometry analysis}

For FISH and IgA-coated bacteria quantification, FCM analysis was performed using a FacsAria SORP sorter (BD, San José, CA, USA) as previously described [5]. Commercial

90 Flow Check ${ }^{\mathrm{TM}}$ Fluorospheres (Beckman Coulter, Inc. FL, USA) were used to determine total

91 counts combined with PI. Analysis was performed using Flowjo v7.6.5 software (Tree Star, 
Inc.). Microbiota composition results are expressed as the $\log _{10}$ of specific probe labeled bacteria counts/g of feces in each sample. Moreover, the Firmicutes to Bacteroidetes $(F / B)$ ratio was calculated taking into account the analyzed bacterial groups belonging to the Firmicutes phylum (those hybridized by Chis150, Erec482, Lab158, Staphy and Strept probes) and those belonging to the Bacteroidetes phylum (those hybridized by the Bac303 probe). IgA-coated bacteria results are expressed as the percentage of bacteria coated with IgA with respect to the total bacteria.

\subsection{Lactic acid and SCFA analysis}

After thawing fecal samples, homogenates were centrifuged to remove any particulate matter. Supernatants were filtered using Millex® filters $(0.22 \mu \mathrm{m}$, Merck Millipore, Darmstadt, Germany). Supernatant $(200 \mu \mathrm{L})$ was added to $50 \mu \mathrm{L}$ of the internal standard (2-ethylbutyric $100 \mathrm{mM}$ in isopropanol) in a Chromacol VALK vial (Thermo Scientific, Langerwehe, Germany) with a Fisher brand adaptor (Fisher Scientific, Loughborough, UK). Each sample was injected into a 1050 series HPLC System (HP, Crawley, West Sussex, UK) equipped with UV detection. The column used was an ion-exclusion REZEX-ROA organic acid column (Phenomenex, Macclesfield, UK) and a SecurityGuard pre-cartridge (Phenomenex) maintained at $85{ }^{\circ} \mathrm{C}$ in a 7981 model oven (Jones Chromatography, Lakewood, USA). Sulfuric acid $(2.6 \mathrm{mM})$ was used as the eluent, and the flow rate was $0.5 \mathrm{~mL} / \mathrm{min}$. Peaks were integrated using Agilent ChemStation software (Agilent Technologies, Oxford, UK).

Quantification of the samples was obtained through calibration curves of lactic, acetic, propionic, butyric and formic acids $(12.5-100 \mathrm{mM})$. Results were expressed as $\mathrm{mM}$ (for total SCFA) and relative increases of the total and individual SCFA with respect to those values found in the RF group. 


\section{2.7. Metagenomics analysis}

118 DNA was extracted from two randomly selected samples from each group using a FastDNA®

119 SPIN Kit (MP Biomedicals, Solon, OH, USA) following the manufacturer's protocol.

120 Amplicons of 16S rDNA were purified and diluted in equal concentrations prior to

121 sequencing in Ion Torrent platforms by the Genetic Diagnostic Bioarray facilities (Bioarray,

122 Alicante, Spain), as previously described [8]. Briefly, a massive sequencing using the QIIME

123 software package v1.8.0. and USEARCH v7.0.1090 was carried out and the obtained

124 sequences were assigned into operational taxonomic units (OTUs; sequences that share $\geq 97 \%$

125 similarity) using the UCLUST algorithm and Greengenes reference database (v13_8). Results

126 are expressed as absolute and relative abundance of phyla and number of detected species.

127 The bacterial species found among the experimental conditions, in common or not, were also

128 considered and represented through a Venn diagram.

\subsection{Statistical analysis}

131 The normality of continuous variables was assessed by normal probability plots and the

132 Shapiro-Wilk test, and the variance equality by Levene's test. Non-normally distributed

133 variables were analyzed by non-parametric tests, specifically Kruskal-Wallis and Mann-

134 Whitney U tests. Normally distributed variables were analyzed by one-way ANOVA followed

135 by Bonferroni post hoc significance test. Student T-test was used to analyze the

136 metagenomics study. $\mathrm{P} \leq 0.05$ was considered statistically significant. Statistical analysis was

137 performed using the software package SPSS 22.0 (IBM Statistical Package for the Social

138 Sciences, version 22.0, Chicago, IL, USA).

139 


\section{$141 \quad$ 3.1. Body weight and food intake}

142 Although the initial body weight was similar among the groups, a statistically slower body

143 weight gain was observed during the study for both the CC and TB groups (Figure 1A). The

144 measurement of the food intake revealed that, even though there was not lower food intake

145 when considering the relative amount per body weight (in all cases it was about $12 \mathrm{~g} / 100 \mathrm{~g}$ of

$146 \mathrm{BW})$, lower absolute food intake per rat in both CC and TB groups than in RF group was

147 found from the first day of diet (Figure 1B).

\subsection{Gut bacterial populations by FISH-FCM}

150 After 15 days of dietary intervention, significant differences in the gut microbiota

151 composition were observed (Figure 2). Concerning total bacteria counts, the CC diet caused

152 the elimination of higher number of bacteria per day than the RF diet. This increase could be

153 associated with the stool amount per day, which was higher in $\mathrm{CC}$ rats $(3.07 \mathrm{~g} \pm 0.11 \mathrm{~g})$ than

154 that from $\mathrm{RF}$ rats $(1.78 \mathrm{~g} \pm 0.10 \mathrm{~g})(\mathrm{P}<0.05)$. Nevertheless, the total bacteria counts relative to

155 fecal weight from $\mathrm{CC}$ fed rats were similar to those in the RF group, whereas the TB group

156 showed lower counts than the other groups $(\mathrm{P}=0.021$ and $\mathrm{P}=0.055$ compared to the $\mathrm{RF}$ and

157 CC groups, respectively).

158 Regarding particular bacterial groups, both the CC and TB groups presented lower counts of

159 E. coli than the RF group, with the counts being even lower in the $\mathrm{CC}$ group than in the TB

160 one. The TB diet also led to significantly lower counts of Bifidobacterium spp., Streptococcus

161 spp. and Clostridium histolyticum-C. perfringens than the RF group. The decrease in the

162 Clostridium group, together with a reduction in the Bacteroidaceae-Prevotellaceae group,

163 was also significant compared to the $\mathrm{CC}$ group. As a result, the Firmicutes counts were lower 
164 in feces from the TB group than those from $\mathrm{RF}$ rats $(\mathrm{P}=0.005)$. Even so, the $F / B$ ratio was not

165 significantly modified in the feces of the studied groups.

166

\subsection{Quantitative metagenomics analysis of gut bacterial populations}

168 After the FISH-FCM analysis of microbiota, a metagenomics approach was carried out in

169 representative feces, in order to get an idea about the most modified species. The

170 metagenomics analysis allowed the relative abundance of the OTUs to be obtained (Figure

$1713)$.

172 The CC group showed a higher proportion of the Firmicutes and a lower proportion of

173 Bacteroidetes phylum members than the RF group, which was associated with a significantly

174 higher F/B ratio than the RF and TB groups. The TB group displayed no changes in

175 Firmicutes and Bacteroidetes phyla but showed a higher proportion of the Tenericutes

176 phylum than the RF and CC groups. A further analysis also revealed changes in the relative

177 abundance of some species (Table 2). Regarding Bacteroidetes phylum, the proportion of the

178 Bacterioidales order and particularly of the Bacteroides genus, e.g. B. acidifaciens, decreased

179 with CC intake, whereas the percentage of the Prevotella genus increased, which was not

180 observed in the TB group. Moreover, in the Cyanobacteria phylum, CC diet led to a higher

181 proportion of the Streptophyta order. With regard to the Firmicutes phylum, CC diet led to a

182 higher proportion of the SHA-98 and Clostridiales order, Butyrivibrio genus

183 (Lachnospiraceae family) and Ruminococcaceae family, and a lower proportion of other

184 Clostridiales (Peptococcaceae family and Anaerotruncus sp.) species. On the other hand, the

185 TB group showed an increase in the proportion of the Erysipelotrichaceae family (Firmicutes

186 phylum), Ralstonia sp. (Proteobacteria phylum) and one bacterium of the Mollicutes class

187 (Tenericutes phylum) (Table 2).

188 


\subsection{Qualitative metagenomics analysis of gut bacterial populations}

190 A total of 71, 80 and 73 different species were detected by metagenomics analysis in feces

191 from the RF, CC and TB groups, respectively (Supplementary Figure 1A). To determine the

192 relation among bacterial species present in each group, a Venn diagram was created

193 (Supplementary Figure 1B). From all the fecal-detected species, 68 were common to all

194 three studied groups. CC intake led to 11 new species; of these, four species were also found

195 in the TB group (species belonging to Bacteroidetes, Firmicutes and Proteobacteria phyla)

196 and seven were exclusively detected in the CC group (including species belonging to the

197 Actinobacteria, Cyanobacteria, Firmicutes and Proteobacteria phyla) (Table 3). Only

198 "Candidatus Arthromitus" (Firmicutes phylum, Clostridia class) was found exclusively in the

199 TB group. Two species were only detected in the RF group, which belonged to the

200 Paraprevotellacea family (Bacteroidetes phylum) and Coprobacillus genus (Table 3). In

201 addition Ruminicoccus flavefaciens (Firmicutes phylum) disappeared in the theobromine-fed

202 animals.

203

$204 \quad 3.5$ Fecal pH, lactic acid and SCFA

205 The TB diet led to higher $\mathrm{pH}$ values than those found after the RF and CC diets (Figure 4A).

206 Fecal concentrations of lactic acid were not significantly affected by the experimental diets

207 (4.26 $\pm 1.54 \mathrm{mM}$ in RF group; $1.96 \pm 0.41 \mathrm{mM}$ in CC group; $2.69 \pm 0.73 \mathrm{mM}$ in TB group).

208 Figure 4B shows the fold-increase of the total and the individual fecal SCFA analyzed

209 (acetic, propionic, butyric and formic acids) in the $\mathrm{CC}$ and TB groups compared to the RF

210 group. The intake of CC and TB led to the detection of significantly higher amounts of total

211 SCFA (sum of acetic, propionic, butyric and formic acid) compared to the RF diet (37.8 \pm

$2123.85 \mathrm{mM}$ and $35.9 \pm 5.98 \mathrm{mM}$ vs $14.5 \pm 8.31 \mathrm{mM}$, respectively). Both CC and TB diets 
213 increased by more than seven times the content of butyric acid compared to the RF diet. The

214 CC diet also led to an increase in acetic acid concentration.

215

\subsection{Percentage of fecal bacteria coated with IgA}

217 The percentage of $\operatorname{IgA-coated}$ bacteria was determined before and at 8 and 15 days of the

218 nutritional intervention (Figure 5). The CC group and, to a lesser extent, the TB group

219 showed lower percentages of fecal IgA-coated bacteria compared to the RF group at days 8

220 and 15.

\section{Discussion}

Cocoa-enriched diets have demonstrated their influence on the gut microbiota and the

224 intestinal immune system, which could be partially attributed to the cocoa's polyphenol and

225 fiber content $[4,5,7,17]$. As far as we are concerned, no data about the effect of theobromine on gut microbiota and immunity have been published before. In the present study, we have established the role of theobromine in the effects of cocoa on gut microbiota composition,

228 SCFA, bacteria coated with $\operatorname{IgA}$ and on body weight increase.

In vitro, in vivo and clinical studies demonstrate that cocoa is able to modulate the growth of gut microbiota $[5,7,15,18]$. Previous studies in rats show that the intake of cocoa-enriched

231 diets for at least three weeks modifies the intestinal microbiota pattern $[5,7,15]$. In the present study, the ingestion of the cocoa diet for two weeks was not able to significantly modify most of the bacterial groups analyzed by FISH-FCM, probably because of the shorter

234 length of this nutritional intervention. However, some changes were observed when

235 theobromine was ingested alone, indicating that theobromine by itself is able to directly or 236 indirectly modify gut microbial populations. The metagenomics analysis, even though it was 
carried out in a small number of samples, allows to have an idea of particular genera and/or

238 species from gut microbiota modified by CC and TB diets and thus, by using both techniques

239 in a complementary manner, we obtained a wider approach of the gut microbiota changes.

240 According our FISH-FCM results, theobromine seems to exert an inhibitory effect on gut

241 microbiota, mainly on bacteria belonging to the Firmicutes phylum (Clostridium histolyticum-

242 C. perfringens group and Streptococcus spp.), Bifidobacterium spp. and E.coli. The effect of

243 TB partially agrees with previously reported effects of a cocoa diet [5, 7] on Clostridium spp.

244 and Streptococcus spp. In addition, according to the metagenomics analysis, the decrease in

245 Firmicutes could be associated with the disappearance of Ruminococcus flavefaciens, a

246 cellulolytic bacterium found to be increased by a flavonoid-enriched diet $[19,20]$. The

247 disappearance of $R$. flavefaciens after the TB diet, although it contained the same cellulose

248 amount as the RF and CC diets, may reflect a particular effect of theobromine on this species

249 that could be counteracted by the flavonoid content in the cocoa diet. Conversely,

250 theobromine alone seems to be able to increase other bacteria from the same family

251 (Erysipelotrichaceae). This family is decreased by a diet rich in flavonoids [21], which would

252 explain the current changes observed only in the TB group. Furthermore, from the two

253 samples analyzed in the TB group, it can be suggested that theobromine ingested alone

254 induced the presence of "Candidatus Arthomitus", another member of the Firmicutes phylum.

255 This is a segmented filamentous bacterium able to induce adaptive immune responses in the

256 gut [22], and it can adhere to the epithelial cells in the ileum and Peyer's patches, contributing

257 to the prevention of the colonization of the enteropathogenic E.coli O103, Salmonella, and

258 others $[23,24]$.

259 The cocoa diet, including theobromine, seems to induce the growth of bacteria belonging to

260 Firmicutes, according to the metagenomics analysis. This increase could be associated with a 
higher relative abundance of one species from the Lachnospiraceae (Butyrivibrio genus) and another from the Ruminococcaceae families, all of them belonging to the Clostridia class.

263 Moreover, the cocoa diet seems to lead to the appearance of new species belonging to the 264 Clostridia class (Dehalobacteriaceae spp., Roseburia faecis and SHA-98 spp), which is in 265 line with the increase of Lachnospiraceae, Clostridiales, and Ruminococcaceae found in pigs 266 fed a grape seed extract [25], and therefore, it could be related to an effect of the cocoa's 267 polyphenol content.

In the results of total Bacteroidetes phylum by FISH-FCM and metagenomics analyses, discrepancies were observed, which could be due to the low representation of bacterial members of this phylum in the first analysis and/or the low sample size in the second one.

271 Nevertheless, the metagenomics analysis allowed us to suggest changes inside this phylum.

272 For example, one species from the Paraprevotellaceae family disappeared with both diets, 273 and the cocoa diet decreased in particular the number of species belonging to the Bacteroidales order (Bacteroides sp. and Bacteroides acidifaciens). The B. acidifaciens has been described to be the predominant bacteria responsible for promoting IgA production in the large intestine [26]. This agrees with our current results regarding IgA-coated bacteria and 277 with previous studies showing lower intestinal IgA with a cocoa diet $[5,17,27]$. On the other 278 hand, CC diet increased the relative abundance of Prevotella sp., which could be due to its polyphenol content since higher numbers in the Prevotella group have been associated with 280 the daily consumption of red wine polyphenols [28].

281 One important finding of our study is that theobromine (both in the $\mathrm{CC}$ and TB groups)

282 lowered the counts of E. coli. This agrees with the reported inhibitory effects of theobromine 283 on Gram-negative bacteria [29], suggesting an inhibitory effect on the growth of potential gut 284 pathogens. This inhibition was enhanced with the CC diet, suggesting the role of polyphenols 
in this effect [30]. In the same phylum, Ralstonia sp. seems to appear due to the CC and TB

286 diets. Ralstonia sp. was formerly included in the Pseudomonas genera, which includes species 287 able to degrade methylxanthines [31, 32]. Therefore, its presence may reflect the adaptation of 288 gut microbiota to diets rich in methylxanthines.

The impact of theobromine on gut microbiota was also patent in the Tenericutes phylum, which increased almost fourfold with theobromine ingested alone. This was associated with a 291 higher number of bacteria belonging to the RF39 order (Mollicutes class). A study reported a 292 similar effect with the ingestion of cocoa for 4 weeks [8]. The absence of effects on 293 Tenericutes with the CC diet suggests that other cocoa compounds delayed the theobromine 294 effect on this phylum.

295 With regard to Actinobacteria, a prebiotic effect of cocoa polyphenols in humans [33] and of 296 cocoa fiber in rats [7] by increasing the counts of Bifidobacterium group has been reported.

297 As TB diet, but not CC diet, decreased the proportion of Bifidobacterium spp., it can be 298 suggested that theobromine is counteracting the prebiotic effects of cocoa fiber. However, the 299 metagenomics results suggested no changes in the relative abundance of Actinobacteria 300 species, either in the TB or CC diet, although it seems that CC diet leads the appearance of 301 one species of the Actinomycetales order. In line with these results, blueberries increased the 302 relative abundance of Actinomycetales order in rats, which allows us to suggest the role of 303 polyphenols in such an effect [34]. Finally, the appearance of one species of the Streptophyta 304 order (Cyanobacteria phylum) with the ingestion of cocoa, in agreement with the reported 305 effect of a CC diet for 4 weeks [8], must be related to the cocoa's polyphenol or fiber content. 306 Nevertheless, the role of such bacteria in the intestinal microbiota remains to be elucidated.

307 Overall, this study reveals the impact of theobromine on gut microbiota. The effects were 308 different depending on whether theobromine was ingested alone or when forming part of 
cocoa, although few common characteristics were found. Some changes observed exclusively

310 in the TB group would have been due to the action of this methylxanthine, which were

311 counteracted by other cocoa compounds, such as fiber and polyphenols. Other changes in the

312 TB group agree with previous results reported with a longer CC diet, suggesting that these

313 other compounds included in the CC diet could delay the TB effect. The modifications

314 exclusively found in the CC group must be related to the cocoa's fiber or polyphenol content.

315 The effect of theobromine on gut microbiota has also been reflected by the changes observed in SCFA in both theobromine-containing diets. The enhanced generation of SCFA was mainly due to the butyric acid. Butyrate is considered the main energy source for colonocytes, and is also important for the regulation of gene expression, the intestinal barrier and the immune system, among others [35, 36]. However, whereas butyric acid increased with both

320 diets, the increase in the proportion of acetic acid was only observed after cocoa ingestion.

321 This disagreement could be due to the fermentation of different substrates with both interventions. After cocoa intake, SCFA would come directly from polyphenol and/or fiber fermentation [7], whereas for the TB diet, changes in the generation of SCFA would be indirectly due to the inhibition of some bacterial populations and thus contribute to enhancing 325 the amount of substrate available for other bacteria. The differential patterns in the SCFA 326 generated support the idea that the ingestion of theobromine alone or as part of cocoa has a different impact on gut microbiota. Furthermore, the unexpected higher fecal $\mathrm{pH}$ when theobromine was ingested alone deserves further studies focusing on microbial metabolites which could explain the observed fecal $\mathrm{pH}$ changes.

The current results evidence that theobromine (both in the TB and CC diets) contributes to the 331 lower proportion of bacteria coated with IgA found after the cocoa diet, in line with previous

332 results $[5,7,15]$. As rats fed the $\mathrm{CC}$ diet even showed a lower proportion of $\operatorname{IgA}$-coated 
333 bacteria, the combination of cocoa polyphenols with theobromine in the CC diet could have 334 an additive or a synergistic effect on reducing their proportion. On the other hand, the effect 335 of cocoa fiber must be discarded because it was associated with an increase in the percentage 336 of IgA-coated bacteria [7].

337 Results regarding body weight suggest that theobromine present in cocoa was the main reason 338 for a slower body weight increase produced by the $10 \%$ cocoa diet. In fact, there was a lower

339 food intake per animal already in the first day of diet, which could affect the body weight 340 increase and it can also influence gut microbiota. On the other hand, body growth could be 341 affected by TB influence on metabolism. In this sense, it has been demonstrated that caffeine 342 has a stimulatory effect on thermogenesis [37] and has been associated with bone mass loss 343 [38].

344 In conclusion, here we demonstrate that cocoa theobromine plays a relevant role in some

345 effects related to cocoa intake, such as lower body weight increase and the proportion of $\operatorname{IgA}$ -

346 coated bacteria. In addition, theobromine modifies gut microbiota, although other cocoa

347 compounds -such as cocoa polyphenols or fiber- also act on the intestinal bacteria,

348 attenuating or enhancing the theobromine effects, that overall leads to the global effect of

349 cocoa on microbiota which differs from that of each particular cocoa component.

\section{Author contributions}

352 The authors' contributions were as follows: À.F., F.J.P.-C. and M.C. conceived and designed 353 the study; M.C.-B. and M.M.-C. were responsible for the animal experiments and sampling;

354 S.M.-P. carried out the FISH-FCM and IgA-coated bacteria analyses; S.M.-P, M.C.-B., 355 M.M.-C., F.J.P.-C. and M.C. carried out the metagenomics data analysis; M.R.-A. analyzed 356 the SCFA; S.M -P. and M.C.-B were mainly responsible for the interpretation of the results 
357 and the writing of the final manuscript; F.J.P.-C and M.C. contributed to the critical revision 358 of the manuscript. All authors have read and approved the final version of the manuscript for 359 publication.

361 Acknowledgments

362 The authors would like to thank the Flow Cytometry and Genomic Services of the 'Centres

363 Científics i Tecnològics' of the University of Barcelona (CCiT-UB) and the Genetic

364 Diagnostic Bioarray facilities (Bioarray, Alicante, Spain) for their technical assistance. We

365 also want to thank Idilia Foods S.L. for providing the conventional cocoa extract. The present

366 study was supported by a grant from the Spanish Ministry of Economy and Competitivity

367 (AGL2011-24279). S.M.-P. was supported by a postdoctoral contract of the ISCIII (Sara

368 Borrell, CD10/00224). M. C.-B. holds a fellowship from the University of Barcelona

369 (APIF2014).

370

371 Disclosures: All authors declare no conflict of interest. None of the funders had a role in the

372 design or analysis of the study or in the writing of this article. 


\section{REFERENCES}

[1] Lippi, D., Chocolate in history: Food, medicine, medi-food. Nutrients 2013, 5, 1573-1584.

[2] Ellam, S., Williamson, G., Cocoa and human health. Annu. Rev. Nutr. 2013, 33, $105-128$.

[3] Pérez-Cano, F.J., Massot-Cladera, M., Franch, A., Castellote, C., et al., The effects of cocoa on the immune system. Front. Pharmacol. 2013, 4, 1-12.

[4] Ramiro-Puig, E., Pérez-Cano, F.J., Ramos-Romero, S., Pérez-Berezo, T., et al., Intestinal immune system of young rats influenced by cocoa-enriched diet. $J$. Nutr. Biochem. 2008, 19, 555-565.

[5] Massot-Cladera, M., Pérez-Berezo, T., Franch, A., Castell, M., et al., Cocoa modulatory effect on rat faecal microbiota and colonic crosstalk. Arch. Biochem. Biophys. 2012, 527, 105-12.

[6] Massot-Cladera, M., Franch, À., Pérez-Cano, F.J., Castell, M., Cocoa and cocoa fibre differentially modulate $\operatorname{IgA}$ and $\operatorname{IgM}$ production at mucosal sites. Br. J. Nutr. 2016, 115, 1539-1546.

[7] Massot-Cladera, M., Costabile, A., Childs, C.E., Yaqoob, P., et al., Prebiotic effects of cocoa fibre on rats. J. Funct. Foods 2015, 19, 341-352.

[8] Camps-Bossacoma, M., Pérez-Cano, F.J., Franch, À., Castell, M., Gut microbiota in a rat oral sensitization model: effect of a cocoa-enriched diet. Oxid. Med. Cell. Longev. 2017, 2017, 1-12.

[9] Katz, D.L., Doughty, K., Ali, A., Cocoa and chocolate in human health and disease. Antioxid. Redox Signal. 2011, 15, 2779-2811.

[10] Franco, R., Oñatibia-Astibia, A., Martínez-Pinilla, E., Health benefits of methylxanthines in cacao and chocolate. Nutrients 2013, 5, 4159-4173.

[11] Srdjenovic, B., Djordjevic-Milic, V., Grujic, N., Injac, R., et al., Simultaneous HPLC determination of caffeine, theobromine, and theophylline in food, drinks, and herbal products. J. Chromatogr. Sci. 2008, 46, 144-149.

[12] Martínez-Pinilla, E., Oñatibia-Astibia, A., Franco, R., The relevance of theobromine for the beneficial effects of cocoa consumption. Front. Pharmacol. 2015, 6, 1-5.

[13] Daneshvar, A., Aboulfadl, K., Viglino, L., Broséus, R., et al., Evaluating pharmaceuticals and caffeine as indicators of fecal contamination in drinking water sources of the Greater Montreal region. Chemosphere 2012, 88, 131139.

[14] Miller, G.E., Radulovic, L.L., DeWit, R.H., Brabec, M.J., et al., Comparative theobromine metabolism in five mammalian species. Drug Metab. Dispos. 1984, 12, 154-160.

[15] Massot-Cladera, M., Abril-Gil, M., Torres, S., Franch, A., et al., Impact of cocoa polyphenol extracts on the immune system and microbiota in two strains 
of young rats. Br. J. Nutr. 2014, 112, 1944-1954.

[16] Magne, F., Hachelaf, W., Suau, A., Boudraa, G., et al., Effects on faecal microbiota of dietary and acidic oligosaccharides in children during partial formula feeding. J. Pediatr. Gastroenterol. Nutr. 2008, 46, 580-588.

[17] Pérez-Berezo, T., Franch, A., Castellote, C., Castell, M., et al., Mechanisms involved in down-regulation of intestinal $\operatorname{IgA}$ in rats by high cocoa intake. $J$. Nutr. Biochem. 2012, 23, 838-844.

[18] Etxeberria, U., Fernández-Quintela, A., Milagro, F.I., Aguirre, L., et al., Impact of polyphenols and polyphenol-rich dietary sources on gut microbiota composition. J. Agric. Food Chem. 2013, 61, 9517-9533.

[19] Venditto, I., Luis, A.S., Rydahl, M., Schückel, J., et al., Complexity of the Ruminococcus flavefaciens cellulosome reflects an expansion in glycan recognition. Proc. Natl. Acad. Sci. 2016, 113, 201601558.

[20] Klinder, A., Shen, Q., Heppel, S., Lovegrove, J.A., et al., Impact of increasing fruit and vegetable and flavonoid intake on the human gut microbiota. Food Funct. 2016, 7, 1788-1796.

[21] Etxeberria, U., Arias, N., Boqué, N., Macarulla, M.T., et al., Reshaping faecal gut microbiota composition by the intake of trans-resveratrol and quercetin in high-fat sucrose diet-fed rats. J. Nutr. Biochem. 2015, 26, 651-660.

[22] Ericsson, A.C., Hagan, C.E., Davis, D.J., Franklin, C.L., Segmented filamentous bacteria: Commensal microbes with potential effects on research. Comp. Med. 2014, 64, 90-98.

[23] Snel, J., Segmented filamentous bacteria and increased resistance to infection, in: Heidt, P.J., Tore, M., Rusch, V., Vesalovic, J. (Eds.), Bacterial Species as Partners and Pathogens, Old Herbon University Foundation, Herborn-Dill, Germany 2012, pp. 63-72.

[24] Heczko, U., Abe, A., Finlay, B.B., Segmented filamentous bacteria prevent colonization of enteropathogenic Escherichia coli $\mathrm{O} 103$ in rabbits. J. Infect. Dis. 2000, 181, 1027-1033.

[25] Choy, Y.Y., Quifer-Rada, P., Holstege, D.M., Frese, S.A., et al., Phenolic metabolites and substantial microbiome changes in pig feces by ingesting grape seed proanthocyanidins. Food Funct. 2014, 5, 2298-2308.

[26] Yanagibashi, T., Hosono, A., Oyama, A., Tsuda, M., et al., IgA production in the large intestine is modulated by a different mechanism than in the small intestine: Bacteroides acidifaciens promotes IgA production in the large intestine by inducing germinal center formation and increasing the number of IgA+ B cell. Immunobiology 2013, 218, 645-651.

[27] Massot-Cladera, M., Franch, A., Castellote, C., Castell, M., et al., Cocoa flavonoid-enriched diet modulates systemic and intestinal immunoglobulin synthesis in adult Lewis rats. Nutrients 2013, 5, 3272-3286.

[28] Queipo-Ortuño, M.I., Influence of red wine polyphenols on the gut microbiota ecology. Am. J. Clin. Nutr. 2012, 95, 1323-1334.

[29] Piddock, L.J. V, Garvey, M.I., Rahman, M.M., Gibbons, S., Natural and 
synthetic compounds such as trimethoprim behave as inhibitors of efflux in Gram-negative bacteria. J. Antimicrob. Chemother. 2010, 65, 1215-1223.

[30] Fathima, A., Rao, J.R., Selective toxicity of catechin - a natural flavonoid towards bacteria. Appl. Microbiol. Biotechnol. 2016, 100, 6395-6402.

[31] Gokulakrishnan, S., Chandraraj, K., Gummadi, S.N., A preliminary study of caffeine degradation by Pseudomonas sp. GSC 1182. Int. J. Food Microbiol. 2007, 113, 346-350.

[32] Summers, R.M., Louie, T.M., Yu, C.L., Subramanian, M., Characterization of a broad-specificity non-haem iron N-demethylase from Pseudomonas putida CBB5 capable of utilizing several purine alkaloids as sole carbon and nitrogen source. Microbiology 2011, 157, 583-592.

[33] Tzounis, X., Rodriguez-Mateos, A., Vulevic, J., Gibson, G.R., et al., Prebiotic evaluation of cocoa-derived flavanols in healthy humans by using a randomized, controlled, double-blind, crossover intervention study. Am. J. Clin. Nutr. 2011, 93, 62-72.

[34] Lacombe, A., Li, R.W., Klimis-Zacas, D., Kristo, A.S., et al., Lowbush wild blueberries have the potential to modify gut microbiota and xenobiotic metabolism in the rat colon. PLoS One 2013, 8, 1-8.

[35] Puertollano, E., Kolida, S., Yaqoob, P., Biological significance of short-chain fatty acid metabolism by the intestinal microbiome. Curr Opin Clin Nutr Metab Care 2014, 17, 1-6.

[36] Leonel, A.J., Alvarez-Leite, J.I., Butyrate: implications for intestinal function. Curr. Opin. Clin. Nutr. Metab. Care 2012, 15, 474-479.

[37] Stohs, S.J., Badmaev, V., A review of natural stimulant and non-stimulant thermogenic agents. Phyther. Res. 2016, 30, 732-740.

[38] Zhou, Y., Guan, X.X., Zhu, Z.L., Guo, J., et al., Caffeine inhibits the viability and osteogenic differentiation of rat bone marrow-derived mesenchymal stromal cells. Br. J. Pharmacol. 2010, 161, 1542-1552. 


\section{FIGURE LEGENDS}

Figure 1. Body weight (A) and food intake (B) throughout the study. The amount of food intake showed in each day was calculated considering the amount fed in each interval divided into the number of days in each period. Values are expressed as mean $\pm \operatorname{SEM}(n=7)$. RF, reference group; $\mathrm{CC}$, group fed diet containing 10\% cocoa; TB, group fed diet containing $0.25 \%$ theobromine. Statistical differences between groups and days of study are shown with different letters.
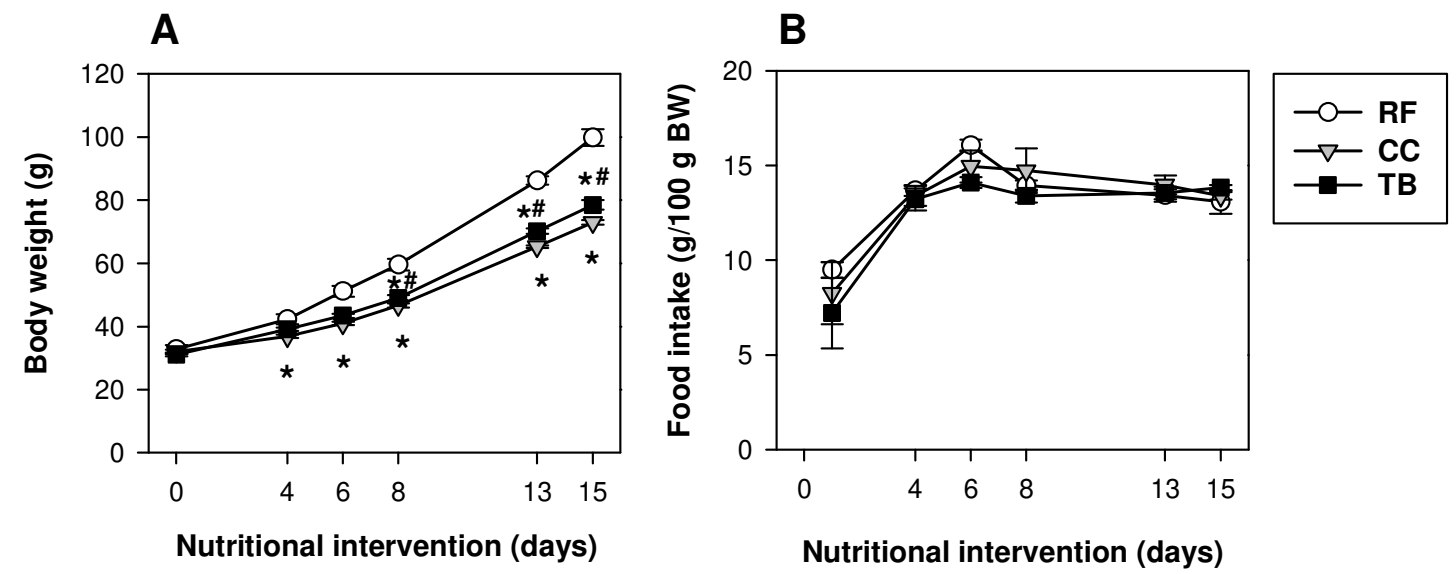
Figure 2: Total bacteria counts, total Firmicutes counts, Firmicutes/Bacteroidetes ratio, and bacteria counts detected with selected probes indicated in the top determined by FISH-FCM from fecal samples. RF, reference group; $\mathrm{CC}$, group fed diet containing $10 \%$ cocoa; $\mathrm{TB}$, group fed diet containing $0.25 \%$ theobromine. A: Actinobacteria, B: Bacteroidetes; F: Firmicutes, P: Proteobacteria. Total bacteria counts are expressed as bacteria/day and bacteria/g feces. Bacterial groups and phylum counts are given as means of $\log _{10}$ bacteria/g feces $\pm \mathrm{SEM}(\mathrm{n}=7)$. * $\mathrm{P}<0.05$ vs $\mathrm{RF}$ group; \# $\mathrm{P}<0.05$ vs $\mathrm{CC}$ group.
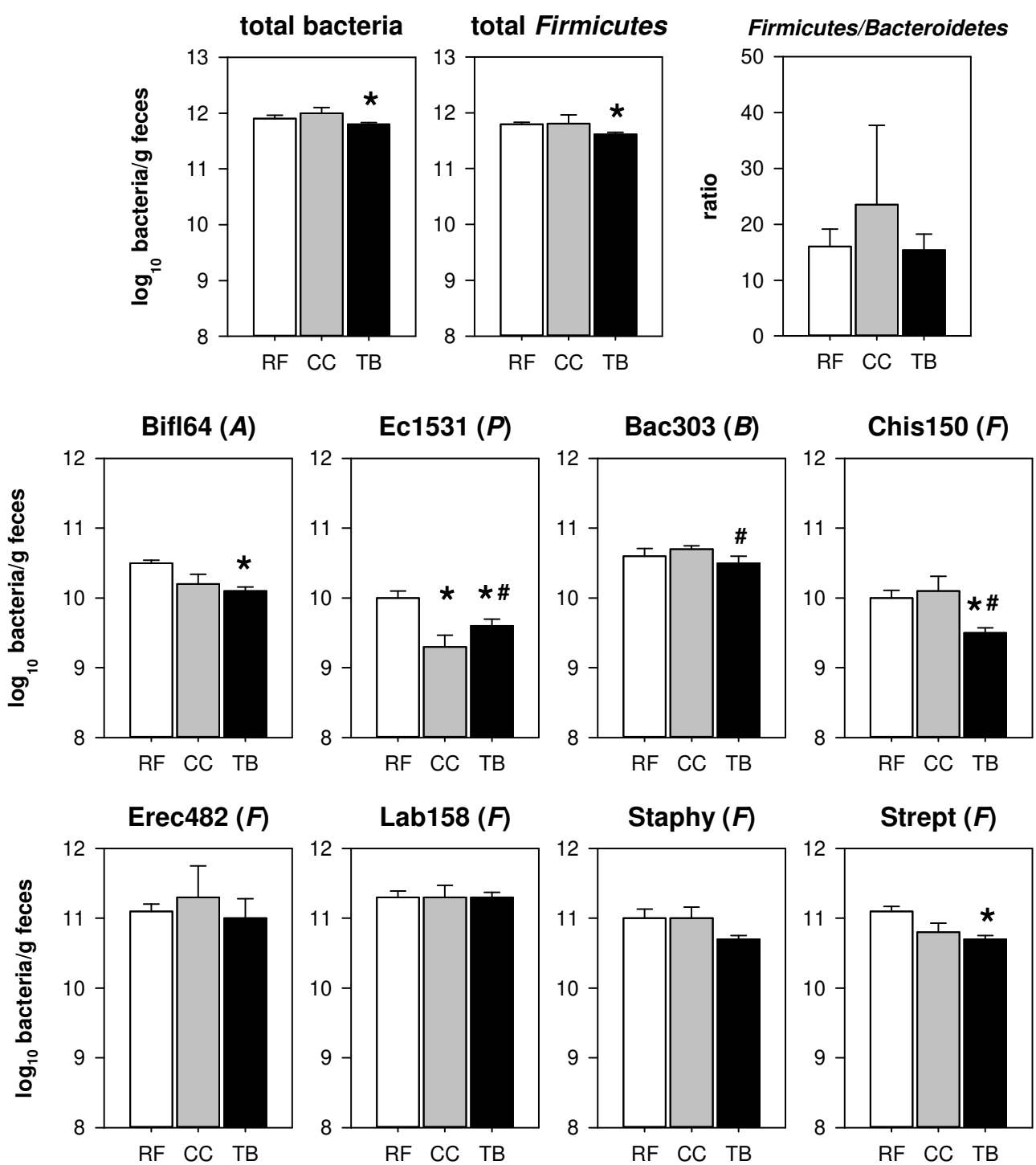
Figure 3. Abundance of phyla found in feces by metagenomics analysis.

Firmicutes/Bacteroidetes ratio and relative abundance (\%) of each phylum with respect to the total bacterial DNA for each experimental group. RF, reference group; CC, group fed diet containing $10 \%$ cocoa; TB, group fed diet containing $0.25 \%$ theobromine. Values are given as means $\pm \operatorname{SEM}(\mathrm{n}=2) . * \mathrm{P}<0.05$ vs $\mathrm{RF}$ group; \# $\mathrm{P}<0.05$ vs $\mathrm{CC}$ group.

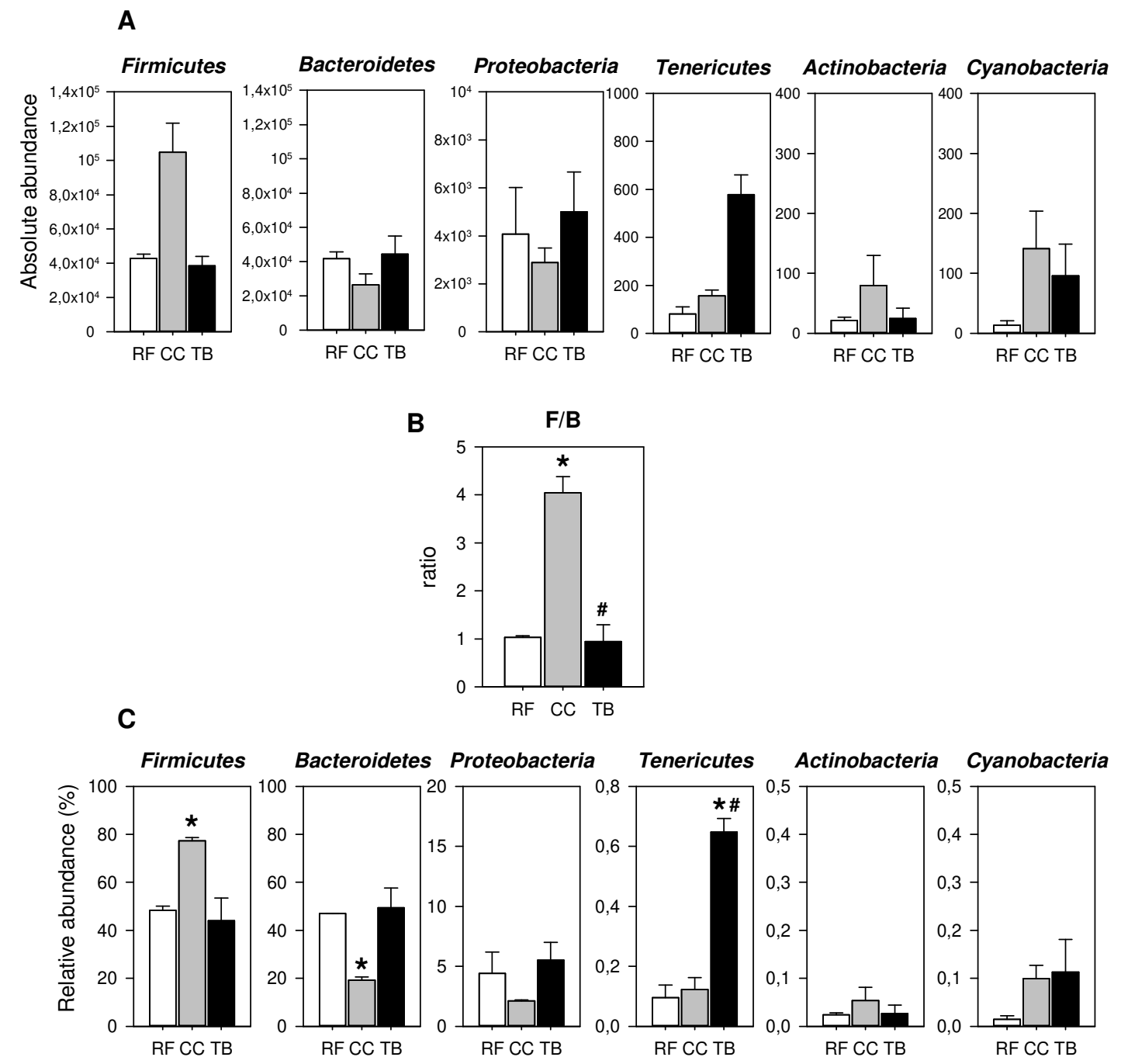


Figure 4. A) Fecal pH. B) Fold change of the total and the individual SCFA analyzed compared to the RF diet which was considered as 1 . Values are expressed as mean \pm SEM $(\mathrm{n}=7)$. RF, reference group; CC, group fed diet containing 10\% cocoa; TB, group fed diet containing $0.25 \%$ theobromine. ${ }^{*} \mathrm{P}<0.05$ vs $\mathrm{RF}$ group; \# $\mathrm{P}<0.05$ vs $\mathrm{CC}$ group.
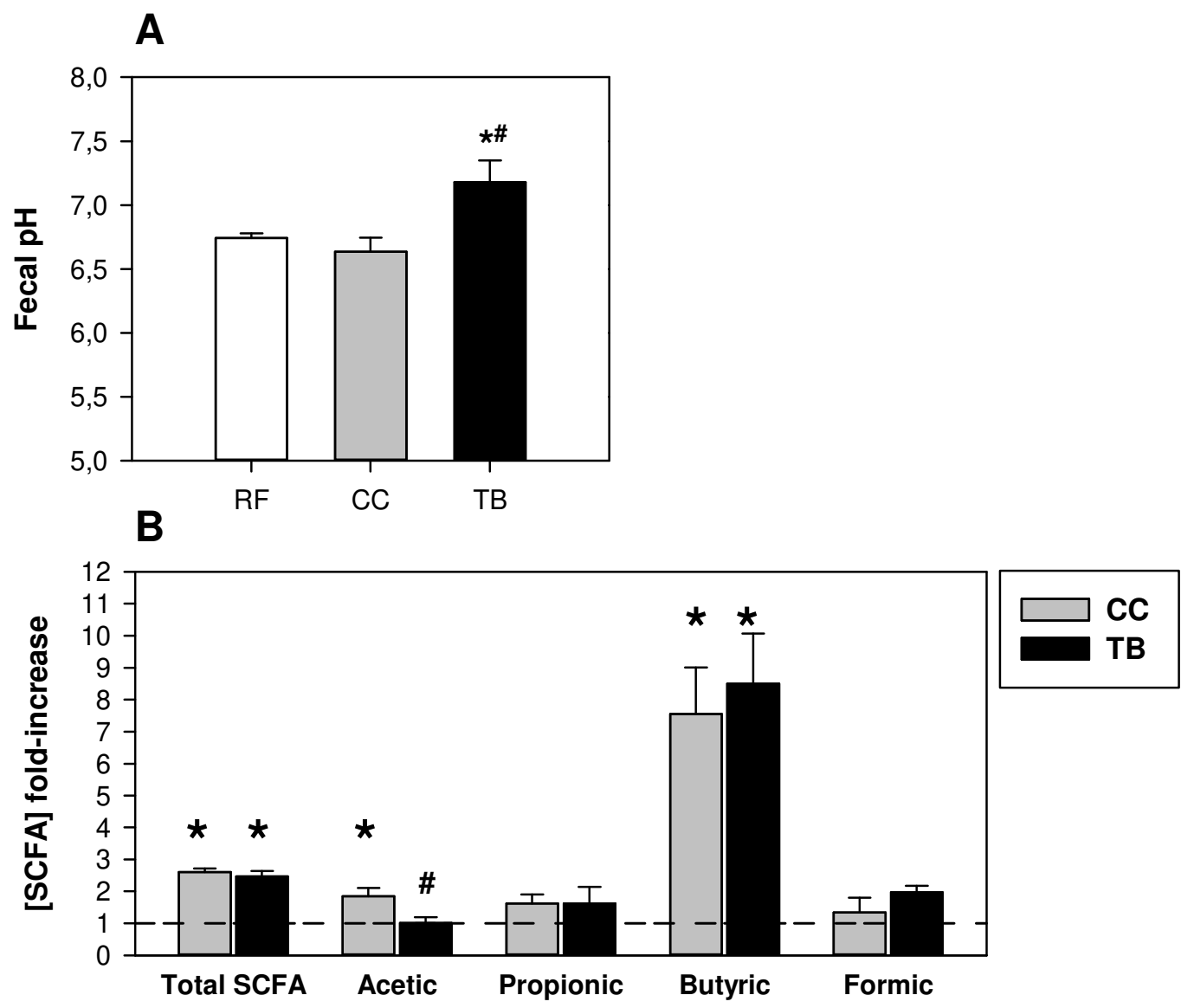
Figure 5. Fecal IgA-coated bacteria throughout the study. Values are expressed as percentage of IgA-coated bacteria (mean \pm SEM, $n=7$ ). RF, reference group; $C C$, group fed diet containing $10 \%$ cocoa; $\mathrm{TB}$, group fed diet containing $0.25 \%$ theobromine. ${ }^{*} \mathrm{P}<0.05$ vs $\mathrm{RF}$ group; \# $\mathrm{P}<0.05$ vs $\mathrm{CC}$ group.

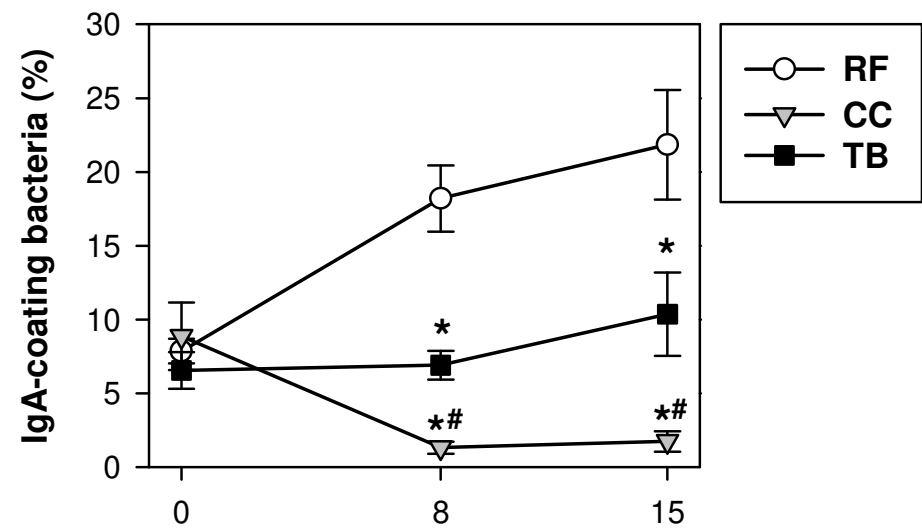

Nutritional intervention (days) 
Table 1. Composition of diets used in the study

\begin{tabular}{lccc}
\hline Components & \multicolumn{3}{c}{ Diets $(\mathbf{g} / \mathbf{k g})^{\mathbf{a}}$} \\
& RF & CC & TB \\
\hline Carbohydrates & 721.9 & 709.5 & 720.1 \\
Proteins & 140.8 & 141.3 & 140.4 \\
Lipids & 38.7 & 38.5 & 38.6 \\
Insoluble fiber & 50.0 & 51.2 & 49.9 \\
Soluble fiber & - & 8.9 & - \\
Micronutrients & 48.6 & 44.1 & 48.5 \\
Theobromine & - & 2.5 & 2.5 \\
Phenolic compounds & - & 4.0 & - \\
\hline Total & $\mathbf{1 0 0 0 . 0}$ & $\mathbf{1 0 0 0 . 0}$ & $\mathbf{1 0 0 0 . 0}$ \\
\hline
\end{tabular}

${ }^{\mathrm{a}} \mathrm{RF}$, reference diet; $\mathrm{CC}$, diet containing $10 \%$ cocoa; $\mathrm{TB}$, diet containing $0.25 \%$ theobromine. 
Table 2: Summary of the results found after analysis of OTU relative abundance in samples belonging to the three studied groups. RF, reference group; CC, group fed diet containing $10 \%$ cocoa; TB, group fed diet containing $0.25 \%$ theobromine. Arrows indicate significant changes $(\mathrm{P}<0.05)$ for each pairwise comparison.

\begin{tabular}{|c|c|c|c|c|c|c|c|}
\hline phylum & class & order & family & $\begin{array}{l}\text { genera } \\
\text { (species) }\end{array}$ & CC vs RF & TB vs RF & TB vs CC \\
\hline \multirow{4}{*}{ Bacteroidetes } & \multirow{4}{*}{ Bacteroidia } & \multirow{4}{*}{ Bacteroidales } & & & $\downarrow$ & $\downarrow$ & \\
\hline & & & \multirow[b]{2}{*}{ Bacteroidaceae } & Bacteroides & $\downarrow$ & & \\
\hline & & & & $\begin{array}{l}\text { Bacteroides } \\
\text { acidifaciens }\end{array}$ & $\downarrow$ & & \\
\hline & & & Prevotellaceae & Prevotella & $\uparrow$ & & $\downarrow$ \\
\hline Cyanobaceria & Chloroplast & Streptophyta & & & $\uparrow$ & & $\downarrow$ \\
\hline \multirow{7}{*}{ Firmicutes } & \multirow{6}{*}{ Clostridia } & \multirow{5}{*}{ Clostridiales } & & & $\uparrow$ & & $\downarrow$ \\
\hline & & & Lachnospiraceae & Butyrivibrio & $\uparrow$ & & $\downarrow$ \\
\hline & & & Peptococcaceae & $r c 4-4$ & $\downarrow$ & & \\
\hline & & & \multirow{2}{*}{ Ruminococcaceae } & & $\uparrow$ & & \\
\hline & & & & Anaerotruncus & $\downarrow$ & & \\
\hline & & SHA-98 & & & $\uparrow$ & & $\downarrow$ \\
\hline & Erysipelotrichi & Erysipelotrichales & Erysipelotrichaceae & & & $\uparrow$ & \\
\hline Preoteobacteria & Betaproteobacteria & Burkholderiales & Oxalobacteraceae & Ralstonia & & $\uparrow$ & \\
\hline Tenericutes & Mollicutes & RF39 & & & & $\uparrow$ & $\uparrow$ \\
\hline
\end{tabular}


Table 3: Bacteria detected in one or two of the studied groups. Grey color indicates bacteria presence. RF, reference group; CC, group fed diet containing $10 \%$ cocoa; TB, group fed diet containing $0.25 \%$ theobromine.

\begin{tabular}{lllll}
\hline Phylum & Class & Order & Family & Genus \\
\hline Bacteroidetes & Bacteroidia & Bacteroidales & Paraprevotellaceae & \\
Firmicutes & Erysipelotrichi & Erysipelotrichales & Erysipelotrichaceae & Coprobacillus \\
Firmicutes & Clostridia & Clostridiales & Ruminococcaceae & Ruminococcus \\
Actinobacteria & Actinobacteria & Actinomycetales & Other & Other \\
Cyanobacteria & Chloroplast & Streptophyta & & \\
Firmicutes & Clostridia & Clostridiales & Dehalobacteriaceae & \\
Firmicutes & Clostridia & Clostridiales & Lachnospiraceae & Roseburia \\
Firmicutes & Clostridia & SHA-98 & & \\
Proteobacteria & Gammaproteobacteria & Aeromonadales & Aeromonadaceae & Other \\
Proteobacteria & Gammaproteobacteria & Pseudomonadales & Pseudomonadaceae & Pseudomona \\
Bacteroidetes & Bacteroidia & Bacteroidales & Prevotellaceae & Prevotella \\
Bacteroidetes & Bacteroidia & Bacteroidales & Prevotellaceae & Prevotella \\
Firmicutes & Bacilli & Bacillales & Staphylococcaceae & Staphylococcus \\
Proteobacteria & Betaproteobacteria & Burkholderiales & Oxalobacteraceae & Ralstonia \\
Firmicutes & Clostridia & Clostridiales & Clostridiaceae & “Candidatus Arthromitus” \\
\hline
\end{tabular}


Supplementary Figure 1. Diversity of bacterial species found in feces by metagenomics analysis. A) Richness of bacterial species; B) Venn diagram of differentially detected species. The diagram shows the absolute number of detected species that belonged to each of the individual nutritional interventions, the detected species common to each pair of groups and the detected species in common to all the three nutritional interventions (in the center of the representation). RF, reference group; CC, group fed diet containing $10 \%$ cocoa; TB, group fed diet containing $0.25 \%$ theobromine.

A
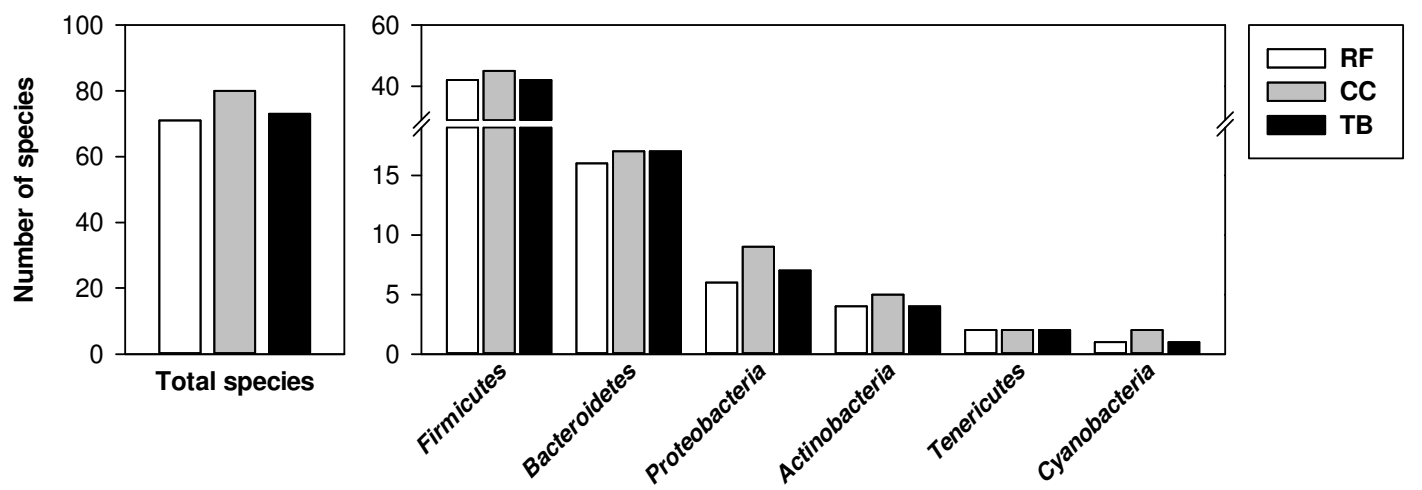

B

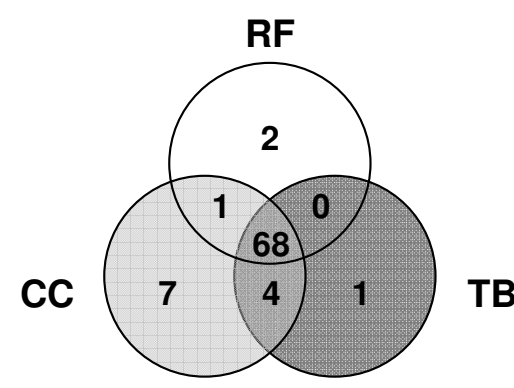


Supplementary Table 1: Bacteria specific probes for the FISH analyses.

\begin{tabular}{lllc}
\hline Bacterial group & Probe & Sequence (5'-3') & References \\
\hline Bacteroidaceae-Prevotellaceae & Bac303 & CCAATGTGGGGGACCTT & {$[1]$} \\
Bifidobacterium spp. & Bif164 & CATCCGGCATTACCACCC & {$[2]$} \\
Clostridium histolyticum-C. Perfringens & Chis150 & TTATGCGGTATTAATCTYCCTTT & {$[3]$} \\
Escherichia coli & Ec1531 & CACCGTAGTGCCTCGTCATCA & {$[4]$} \\
Clostridium coccoides-Eubacterium rectale & Erec482 & GCTTCTTAGTCARGTACCG & {$[5]$} \\
Lactobacillus-Enterococcus & Lab158 & GGTATTAGCAYCTGTTTCCA & {$[6]$} \\
Staphylococcus spp. & Staphy & TCCTCCATATCTCTGCGC & {$[7]$} \\
Streptococcus spp. & Strept & CACTCTCCCCTTCTGCAC & {$[7]$} \\
\hline
\end{tabular}

$Y=(C / T), R=(A / G)$

[1] Manz, W., Amann, R., Ludwig, W., Vancanneyt, M., et al., Application of a suite of $16 \mathrm{~S}$ rRNA-specific oligonucleotide probes designed to investigate bacteria of the phylum cytophaga-flavobacter-bacteroides in the natural environment. Microbiology 1996, 142, $1097-1106$.

[2] Langendijk, P.S., Schut, F., Jansen, G.J., Raangs, G.C., et al., Quantitative fluorescence in situ hybridization of Bifidobacterium spp. with genus-specific 16S rRNA-targeted probes and its application in fecal samples. Applied and Environmental Microbiology 1995, 61, 30693075 .

[3] Harmsen, H.J., Gibson, G.R., Elfferich, P., Raangs, G.C., et al., Comparison of viable cell counts and fluorescence in situ hybridization using specific rRNA-based probes for the quantification of human fecal bacteria. 2000, 183, 125-129.

[4] Poulsen, L.K., Lan, F., Kristensen, C.S., Hobolth, P., et al., Spatial distribution of Escherichia coli in the mouse large intestine inferred from rRNA in situ hybridization. Infection and Immunity 1994, 62, 5191-5194.

[5] Lay, C., Sutren, M., Rochet, V., Saunier, K., et al., Design and validation of $16 \mathrm{~S}$ rRNA probes to enumerate members of the Clostridium leptum subgroup in human faecal microbiota. Environmental Microbiology 2005, 7, 933-946.

[6] Harmsen, H.J.M., Elfferich, P., Schut, F., Welling, G.W., A 165 rRNA-targeted probe for detection of lactobacilli and enterococci in faecal samples by fluorescent in situ hybridization. Microbial Ecology in Health and Disease 1999, 11, 3-12.

[7] Trebesius K, Leitritz L, Adler K, Schubert S, Autenrieth IB, H.J., Culture independent and rapid identification of bacterial pathogens in necrotising fasciitis and streptococcal toxic shock syndrome by fluorescence in situ hybridisation. Med Microbiol Immunol 2000, 188, 169-175. 
Supplementary Table 2: Bacteria detected in one or two of the studied groups. Grey color indicates bacteria presence. RF, reference group; CC, group fed diet containing $10 \%$ cocoa; TB, group fed diet containing $0.25 \%$ theobromine.

\begin{tabular}{|c|c|c|c|c|c|c|}
\hline Phylum & Class & Order & Family & Genus & Specie & RF CC TB \\
\hline Bacteroidetes & Bacteroidia & Bacteroidales & Paraprevotellaceae & & & \\
\hline Firmicutes & Erysipelotrichi & Erysipelotrichales & Erysipelotrichaceae & Coprobacillus & & \\
\hline Firmicutes & Clostridia & Clostridiales & Ruminococcaceae & Ruminococcus & flavefaciens & \\
\hline Actinobacteria & Actinobacteria & Actinomycetales & Other & Other & Other & \\
\hline Cyanobacteria & Chloroplast & Streptophyta & & & & \\
\hline Firmicutes & Clostridia & Clostridiales & Dehalobacteriaceae & & & \\
\hline Firmicutes & Clostridia & Clostridiales & Lachnospiraceae & Roseburia & faecis & \\
\hline Firmicutes & Clostridia & SHA-98 & & & & \\
\hline Proteobacteria & Gammaproteobacteria & Aeromonadales & Aeromonadaceae & Other & Other & \\
\hline Proteobacteria & Gammaproteobacteria & Pseudomonadales & Pseudomonadaceae & Pseudomona & & \\
\hline Bacteroidetes & Bacteroidia & Bacteroidales & Prevotellaceae & Prevotella & Other & \\
\hline Bacteroidetes & Bacteroidia & Bacteroidales & Prevotellaceae & Prevotella & & \\
\hline Firmicutes & Bacilli & Bacillales & Staphylococcaceae & Staphylococcus & Other & \\
\hline Proteobacteria & Betaproteobacteria & Burkholderiales & Oxalobacteraceae & Ralstonia & & \\
\hline Firmicutes & Clostridia & Clostridiales & Clostridiaceae & Candidatus Arthromitus & & \\
\hline
\end{tabular}

